\title{
Analysis of Aero-Thermodynamic Behavior of EXPERT Capsule in Transitional Regime
}

\author{
Gennaro Zuppardi ${ }^{*}$, Luigi Morsa ${ }^{*}$, Antonio Schettino ${ }^{* *}$ and Raffaele Votta ${ }^{* *}$ \\ * Department of Aerospace Engineering - University of Naples "Federico II", \\ Piazzale Tecchio 80, 80125 Naples, Italy \\ ${ }^{* *}$ CIRA, Italian Aerospace Research Center, Via Maiorise, 81043 Capua, Italy
}

\begin{abstract}
The aerodynamic behavior of the EXPERT capsule has been already widely studied at low altitudes. In order to broaden the aerodynamic data base of the capsule, additional computations of the aerodynamic forces and an evaluation of the longitudinal stability and fluctuation of the pressure center have been carried out in the altitude interval 80-105 km. The effect of the rolling angle has been also evaluated. As EXPERT, in the considered altitude interval is in transitional regime, computations have been made by the DSMC code DS3V. Heat flux along the capsule surface has been also evaluated. This is an important topic because the nose and the frustum are made of low and high catalyticity materials, respectively. Computations, already performed in continuum regime by the CFD code H3NS, showed that, at the nose-frustum junction, an abrupt and strong peak of heat flux is present. In this work, this problem has been analyzed also in transitional regime. For this application, the DSMC 2-D code DS2V, requiring smaller computer resources, compared with the ones required by DS3V, has been used for making computations at lower altitudes. Furthermore, using DS2V made possible also to get a more detailed definition of the body surface and therefore to increase the surface resolution. The launch of the capsule is currently scheduled in 2011; flight data should be available to verify the results of the present computations.
\end{abstract}

Keywords: Aerodynamics of the EXPERT capsule, DSMC method, CFD

PACS: 47.45.-n, 47.40.Ki, 47.11.Mn, 47.11.Bc

\section{INTRODUCTION}

EXPERT (European eXPErimental Reentry Testbed) capsule, funded by ESA and supported by a number of European research centers, was designed to enhance knowledge of aero-thermo-dynamic problems during the reentry. Experiments will be carried out about phenomena like gas-surface interaction, induced and natural laminarturbulent transition, real gas effects on shock wave boundary layer interaction, shock layer chemistry and so on. EXPERT will be transported by a Russian Volna rocket at an altitude of about $105 \mathrm{~km}$ and then released. The rocket will be launched from a submarine in the Pacific Ocean.

A number of papers have been already written about EXPERT with different aims, from the evaluation of the aerodynamic behavior to the description of tests and experiments to be made during the re-entry (see [1] and related references). Preliminary computations of aero-thermo-dynamic data base at high altitudes were provided by approximate engineering methods or bridging formulae [1,2]. The aim of the present work is making an additional analysis and, hopefully, a better characterization of the aero-thermo-dynamic data base in rarefied regime.

The present work is focused on the analysis of the aero-thermo-dynamic behavior of the capsule in high altitude flight, between 65 and $105 \mathrm{~km}$. As shown later, EXPERT is in transitional regime in the altitude interval 80-105 km and is in continuum low density regime in the altitude interval $65-80 \mathrm{~km}$. In the altitude interval $80-105 \mathrm{~km}$, the computations are performed using a DSMC approach by means of the "sophisticated" and advanced DS3V code [3]; at each altitude the range of angle of attack is $0-60$ deg.. Furthermore, as the presence of four open flaps makes the flow field, at angle of attack, depending on the rolling angle $\varphi$, the effect of $\varphi$ is evaluated by computations both at $\varphi=0$ and $\varphi=45$ deg.. The analysis will focus on global aerodynamic coefficients, on longitudinal stability and on fluctuation of the position of pressure center.

The heat flux along the capsule surface is also evaluated. To this aim, it is important to consider that the nose and the frustum are made of low and high catalyticity materials, respectively. A number of computations, already performed in continuum [1] by the CFD code H3NS [4], used in 2-D mode, showed that at the nose-frustum 
junction an abrupt and strong peak of heat flux is present. In this paper, heat flux has been computed in transitional regime. For this application the DSMC, 2-D code DS2V [5], requiring smaller computer resources than the ones of DS3V, has been used for making computations at lower altitudes. Furthermore, using DS2V, makes possible also to get a more detailed definition of the body surface and therefore to increase the surface resolution, including the definition of the nose stagnation point. Like DS3V, also DS2V is a sophisticated and advanced code. The computations of heat flux along the capsule surface are made in the altitude interval 70-105 km by DS2V as well as in the interval 65-75 km by H3NS. The results are compared and discussed at the overlapping altitude of $70 \mathrm{~km}$. The launch of the capsule is currently scheduled in 2011; flight data should be available to verify the results of the present computations.

\section{GEOMETRY AND RE-ENTRY TRAJECTORY OF EXPERT}

Figure 1 shows the current baseline geometry of the version $4.4 \mathrm{C}$ of EXPERT. It is a blunted pyramidal shape, consisting of a body of revolution with an ellipse-clothoid-cone 2-D longitudinal profile, the angle of the cone is 12.5 deg.. The total length of the capsule is $1.55 \mathrm{~m}$, the base diameter (D) is $0.918 \mathrm{~m}$. The nose has a local radius of $0.6 \mathrm{~m}$ and an eccentricity of 2.5. The center of mass lies on the axis and is located at $0.868 \mathrm{~m}$ from the nose. The longitudinal profile is cut by 4 planes at an angle of 8.35 deg. to the axis of symmetry. Each plane is equipped with an open flap. Each flap, width $0.4 \mathrm{~m}$ and projected length $0.3 \mathrm{~m}$, is deflected by 20 deg. with respect to the related plane. Finally, it must be pointed out that the fore part of EXPERT ( $x=0.40 \mathrm{~m})$, or nose, is made of low catalytic material (C-SiC). On the contrary, the frustum is made of high catalytic material (PM1000).

As reported by Ivanov [2], the reference surface and the reference length, necessary for reducing the forces and the pitching moment to the related coefficients, are $1.1877 \mathrm{~m}^{2}$ and $1.55 \mathrm{~m}$, respectively. The nominal angle of attack $(\alpha)$ is between 0 and 5 deg. and the re-entry angle is 5.5 deg. [1].

In the altitude interval here considered $(65.2-104.5 \mathrm{~km})$, velocity does not change strongly, ranging from 5038 to $4992 \mathrm{~m} / \mathrm{s}$ [1], the Mach number ranges from 16.1 to 17.0 (the maximum value of 18.3 is met at $\mathrm{h}=88 \mathrm{~km}$ ). The free stream Reynolds $\left(\operatorname{Re}_{\mathrm{D} \infty}\right)$ and Knudsen $\left(\mathrm{Kn}_{\mathrm{D} \infty}\right)$ numbers range from $7.3 \times 10^{4}$ to 82 and from $3.2 \times 10^{-4}$ to $3.1 \times 10^{-1}$, respectively. Figure 1 shows the re-entry trajectory.

EXPERT is in transitional regime in the altitude interval $80.4-104.5 \mathrm{~km}$, where $\mathrm{Kn}_{\mathrm{D} \infty}$, ranges from $4.8 \times 10^{-3}$ to $3.1 \times 10^{-1}$. According to Moss [6], a general definition of the transitional regime is: $10^{-3}<\mathrm{Kn}_{\infty}<50$. The capsule in

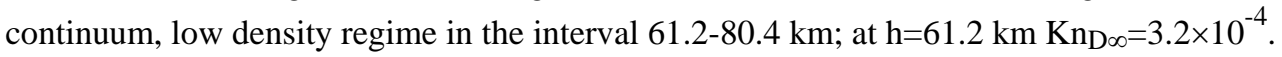

\section{COMPUTING CODES}

\section{Direct Simulation Monte Carlo (DSMC)}

As the DSMC method [7] is supposed to be well known, it will be not described here. On the contrary, some important characteristics of the DSMC codes, DS3V [3] and DS2V [5], used for the present application, will be highlighted in order to point out the reliability of the results. Both codes are able to consider air as a built-in gas. Air is considered made up of five chemical species: $\mathrm{O}, \mathrm{N}, \mathrm{O}_{2}, \mathrm{~N}_{2}$ and $\mathrm{NO}$ in thermo-chemical non equilibrium. The built-in chemical model relies on 23 chemical reactions.

Both codes are "sophisticated" and advanced. A DSMC code is defined sophisticated [8] if: i) it uses two sets of cells (collision and sampling cells) with the related cell adaptation, ii) it implements procedures promoting nearest neighbor collisions, iii) it generates automatically computational parameters such as number of cells and number of simulated molecules by the input number of megabytes, iv) it provides optimal values of the time step. Besides being sophisticated, DS3V and DS2V are also advanced because allowing the user to evaluate the quality of a run in terms of the adequacy of the number of simulated molecules by the "on line", i.e. during the run, visualization of the ratio of the molecule mean collision separation (mcs) and the mean free path ( $\lambda$ ), in the same cell; mcs/ $\lambda$ should be 
less than unity everywhere in the computational domain. Bird [3, 5] suggests 0.2 as a limit value for an optimal quality of the run.

\section{Computational Fluid-Dynamics (CFD)}

The CFD code H3NS [4], developed at the CIRA Aero-thermo-dynamics and Space Propulsion Laboratory, solves full Reynolds averaged Navier-Stokes equations and, like DS2/3V codes, considers air made up of the five above mentioned chemical species in thermo-chemical non-equilibrium. The Park model [9], using 17 chemical reactions, is implemented. The reactions are the same like the ones implemented in the DS2/3V codes, but the Park model is lacking of 2 exchange reactions $\left(\mathrm{O}_{2}+\mathrm{N} \rightarrow \mathrm{NO}+\mathrm{O}, \quad \mathrm{NO}+\mathrm{N} \rightarrow \mathrm{N}_{2}+\mathrm{O}\right)$ and 4 recombination reactions $\left(\mathrm{O}+\mathrm{O}+\mathrm{O}_{2} \rightarrow \mathrm{O}_{2}+\mathrm{O}_{2}, \mathrm{~N}+\mathrm{N}+\mathrm{N}_{2} \rightarrow \mathrm{N}_{2}+\mathrm{N}_{2}, \mathrm{~N}+\mathrm{N}+\mathrm{O} \rightarrow \mathrm{N}_{2}+\mathrm{O}, \mathrm{N}+\mathrm{O}+\mathrm{N} \rightarrow \mathrm{NO}+\mathrm{N}\right)$.

The code is based on a finite volume approach with a cell-centered formulation. The inviscid fluxes are computed by a flux difference splitting scheme. Second-order approximation is obtained with an essentially non oscillatory reconstruction of interface values. The runs are performed by an explicit multistage Runge-Kutta algorithm, coupled with an implicit evaluation of the source terms.

In order to take into account the effects of rarefaction, H3NS can implement, as boundary conditions, slip velocity $\left(V_{S}\right)$ and slip temperature $\left(T_{S}\right)$. Among the many available formulations of this kind of conditions, the ones proposed by Kogan [10] are used:

$$
\mathrm{V}_{\mathrm{s}}=1.012 \lambda\left(\frac{\partial \mathrm{u}}{\partial \mathrm{n}}\right)_{\mathrm{w}} \quad \text { and } \quad \mathrm{T}_{\mathrm{s}}=\mathrm{T}_{\mathrm{W}}+1.73 \frac{\gamma}{\gamma-1} \frac{\sqrt{\pi}}{4} \lambda\left(\frac{\partial \mathrm{T}}{\partial \mathrm{n}}\right)_{\mathrm{W}}
$$

where: $\mathrm{u}$ is the velocity tangential component, $\gamma$ is the ratio of specific heat at constant pressure and volume, $\mathrm{n}$ is the local normal and $\mathrm{w}$ is for wall.

\section{TEST CONDITIONS}

The computational domain of DS3V was a parallelepiped: $\mathrm{x}=2.4 \mathrm{~m}, \mathrm{y}=2.3 \mathrm{~m}, \mathrm{z}=1.1 \mathrm{~m}$. The computational domain of DS2V was a rectangle in the meridian plane: $x=2.4 \mathrm{~m}$ and $\mathrm{y}=0.3 \mathrm{~m}$. For all 3-D and 2-D runs, simulation time was longer than 25 times the time necessary to cross the computing region along the $\mathrm{x}$ direction at the free stream velocity $\left(\cong 5 \times 10^{-4} \mathrm{~s}\right)$. This simulation time can be considered long enough for stabilizing all thermo-aerodynamic parameters. The number of simulated molecules was about $2.0 \times 10^{7}$ for both $2-\mathrm{D}$ and $3-\mathrm{D}$ tests. This number of molecules provided: for 3-D tests, at the most severe test condition of $80.4 \mathrm{~km}$, an average value of mcs/ $\lambda$ of about 1.1 , for 2-D tests, at the most severe test conditions of $69.8 \mathrm{~km}$, an average value of mcs $/ \lambda$ of about 0.25 ; thus both 3-D and 2-D results at these altitudes are not fully reliable.

The computation domain of H3NS was a grid of 5200 cells (10812 points), following the shock wave profile. The grid was obtained with subsequent thickening until the solution did not stabilize, i.e. did not show any variation.

All aerodynamic tests by DS3V were made in the range of angle of attack 0-60 deg. with a step of 5 deg.. The aerodynamic forces were evaluated on the assumption of non-reactive surface. On the opposite, according to what above said, the computation of heat flux relies on the conservative hypothesis, not far from reality, of non-reactive surface for the nose and of fully catalytic surface for the frustum.

The surface recombination reactions, implemented both in DS2V and H3NS were: $\mathrm{O}+\mathrm{O} \rightarrow \mathrm{O}_{2}, \mathrm{~N}+\mathrm{N} \rightarrow \mathrm{N}_{2}$ and $\mathrm{N}+\mathrm{O} \rightarrow \mathrm{NO}$. For the computation of both aerodynamic forces and heat flux, the wall temperature was $300 \mathrm{~K}$. This temperature, pretty low in a re-entry analysis, was chosen to get more conservative values of heat flux. Free stream thermodynamic parameters were provided by the U.S. Standard Atmosphere 1976.

\section{ANALYSIS OF THE RESULTS}

The high altitude, aerodynamic behavior of EXPERT is shown in Figs.2a, b where the profiles of the axial $\left(C_{A}\right)$ and the normal $\left(\mathrm{C}_{\mathrm{N}}\right)$ force coefficients are reported as functions of the angle of attack $\alpha$, in the altitude interval 80.4$104.5 \mathrm{~km}$. Most of computations have been made with $\varphi=0$. In order to evaluate the influence of the rolling angle, only two sets of tests with $\varphi=45 \mathrm{deg}$. have been made at $\mathrm{h}=80.4$ and $\mathrm{h}=104.5 \mathrm{~km}$. Figures show that, in this altitude interval, the influence of $\varphi$ is negligible. Very slight difference is detectable at high angle of attack (say $\alpha>40$ deg.), therefore, considering that, as said before, the angle of attack of EXPERT is not higher than 5 deg., the influence of the rolling angle is practically irrelevant. 


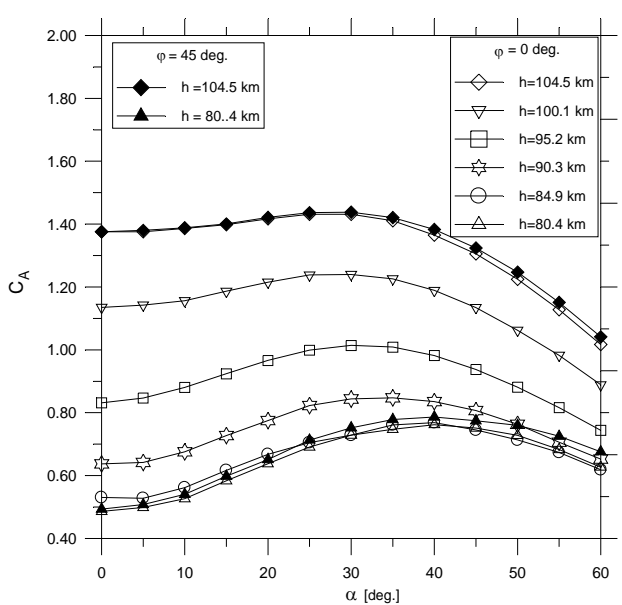

(a)

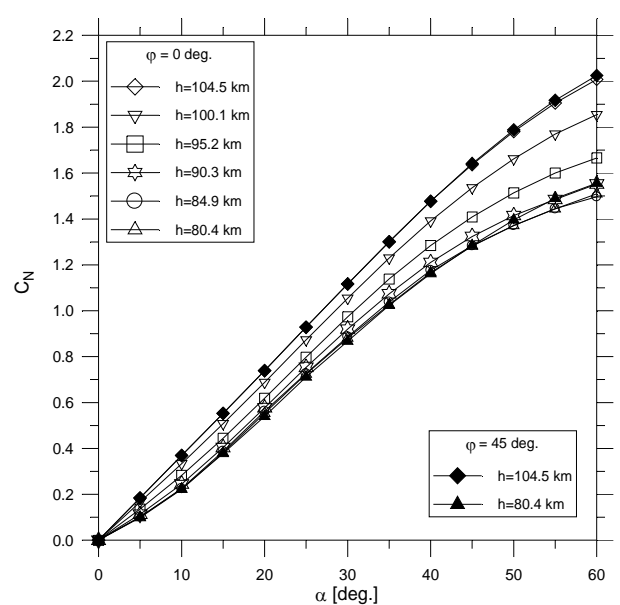

(b)

Figure 2. Profiles of axial (a) and normal (b) force coefficients of EXPERT in the altitude interval 80.4-104.5 km

The capsule is longitudinally stable. A measure of stability is provided by the profiles of the pitching moment around the gravity center $\left(\mathrm{C}_{\mathrm{Mcg}}\right)$ and by the location of the pressure center $\left(\mathrm{x}_{\mathrm{cp}}\right)$ along the axis, reported in Figs.3a, $\mathrm{b}$, respectively. The longitudinal equilibrium $\left(\mathrm{dC}_{\mathrm{Mcg}} / \mathrm{d} \alpha<0\right)$, with a trim angle up to about 40 deg., is kept at all altitudes. The pressure center is, at least, at $0.14 \mathrm{~m}$ behind the center of gravity $\left(\mathrm{x}_{\mathrm{cg}}\right)$. Also the longitudinal stability and the position of the pressure center practically are not influenced by the rolling angle.

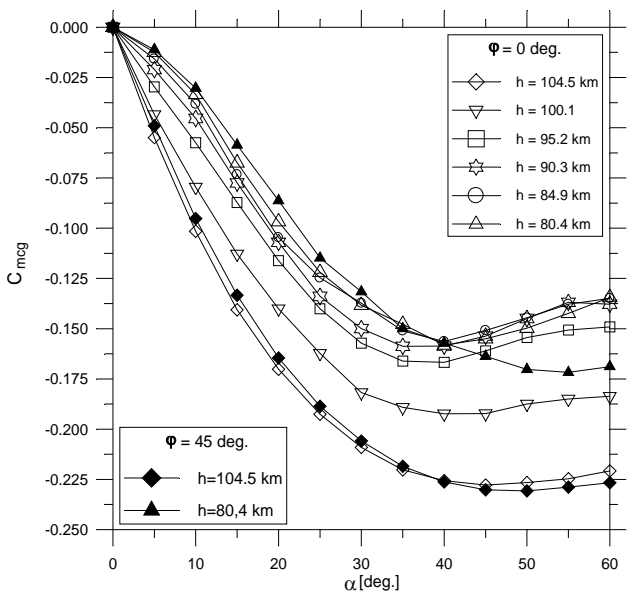

(a)

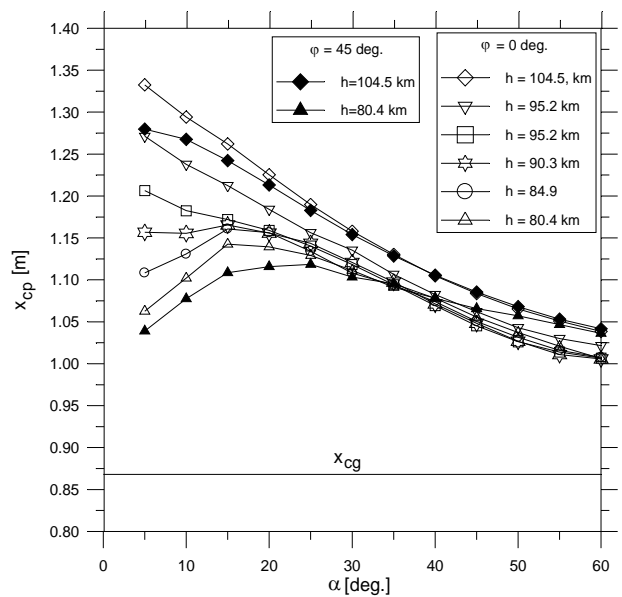

(b)

Figure 3. Profiles of pitching moment coefficient (a) and position of pressure center along the axis of EXPERT in the altitude interval 80.4-104.5 km

Figure 4a shows the profiles of heat flux along the capsule surface in the altitude interval 69.8-104.5 km by DS2V. All heat flux profiles, along the capsule surface, show an abrupt decrease at the junction of nose-frustum $(\mathrm{x}=0.40 \mathrm{~m})$, where a step of $4 \mathrm{~mm}$ is present, producing an aerodynamic shadow. Even though the nose surface has been considered non-reactive while the frustum surface is fully catalytic, the peak of heat flux on the flap is much lower than the one at the nose stagnation point. For example, at the altitudes of 69.8 and $104.5 \mathrm{~km}$, heat flux at the nose stagnation point are $3.59 \times 10^{5}$ and $1.26 \times 10^{4} \mathrm{~W} / \mathrm{m}^{2}$, while the peak of heat flux on the flap are $5.89 \times 10^{4}$ and $4.41 \times 10^{3} \mathrm{~W} / \mathrm{m}^{2}$, respectively; the ratio of the two heat fluxes ranges from 6 and 3 . The effect of catalyticity (nose non catalytic and frustum catalytic) is not easily detectable from Fig.4a. For this reason, in order to show the effect of catalyticity, Fig.4b reports, as typical example, a blow up of the heat flux profile, at $\mathrm{h}=69.8 \mathrm{~km}$ along the frustum, considering this part of surface fully catalytic and non-reactive. 


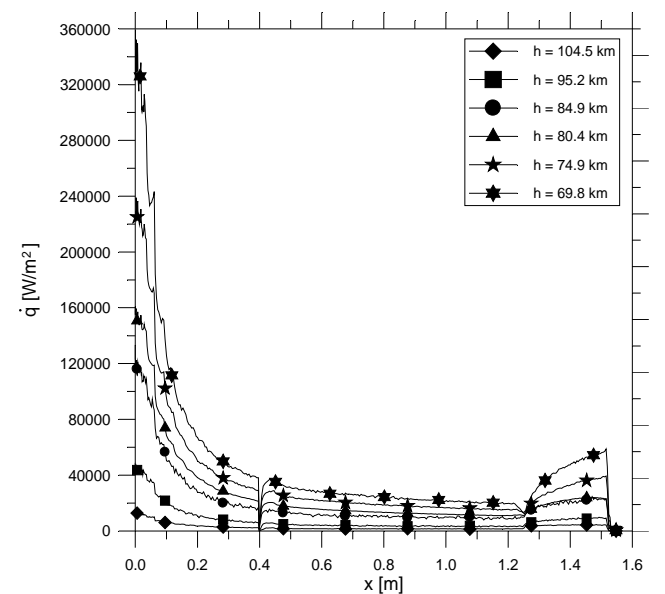

(a)

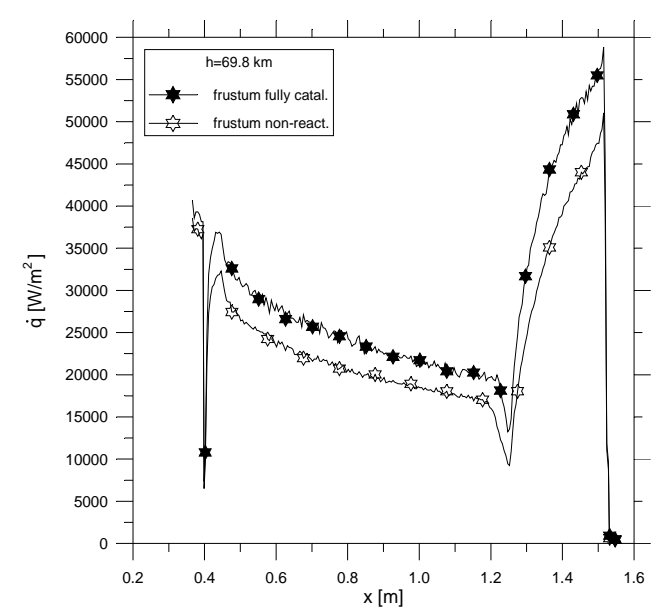

(b)

Figure 4. Profiles of heat flux along the EXPERT surface in the altitude interval 69.8-104.5 km (a), comparison of heat flux profiles along the frustum, at $\mathrm{h}=80.4 \mathrm{~km}$, considering the surface fully catalytic and non-reactive (b)

A comparison of heat flux computed by DS2V with the one computed by H3NS is shown in Fig.5a, b where heat fluxes are reported along the surface at $\mathrm{h}=69.8 \mathrm{~km}$ and at the nose stagnation point in the altitude interval 65.2-84.9 $\mathrm{km}$. In order to avoid unwanted fluid-dynamic influence, produced by the step at the junction nose/frustum, both runs have been carried out without considering the step and, in order to highlight the difference between the DSMC and CFD methods, H3NS was run without including slip corrections. The main difference between the two methods is an abrupt increase of heat flux by H3NS at the junction nose/frustum (Fig.5a). As shown later, this is probably due to different chemical compositions evaluated by the two codes.

Computations of heat flux at the nose stagnation point (Fig.5b) have been carried out considering the surface of the nose both non-reactive and fully catalytic. As expected, the influence of catalyticity increases with decreasing altitude; this is due to an increase of probability of dissociation and therefore of recombination on the capsule surface. Catalyticity produces, in the altitude interval 84.9-69.8 km, a percentage increase of the nose stagnation point heat flux by DS2V between 2.7\%-21.2\%. Catalyticity produces, in the altitude interval 74.9-65.2 km, a percentage increase of the nose stagnation point heat flux by H3NS between $66.2 \%-89.8 \%$. Heat fluxes computed by H3NS are always lower than the ones computed by DS2V; both for non-reactive and for fully catalytic surface.

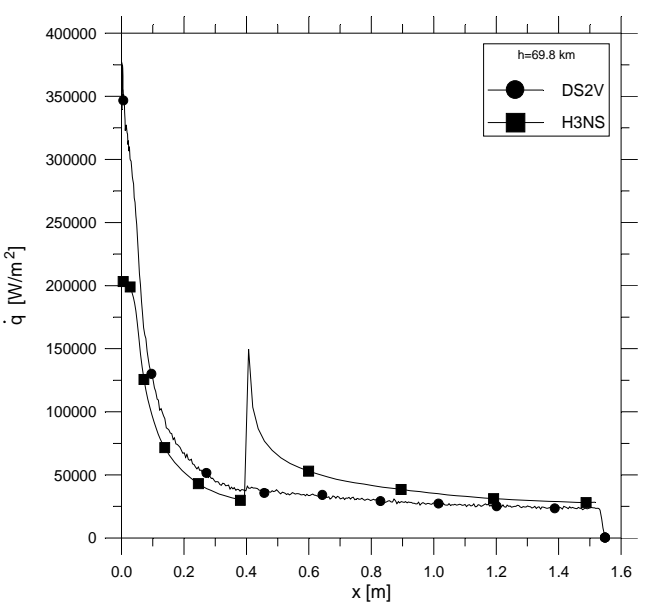

(a)

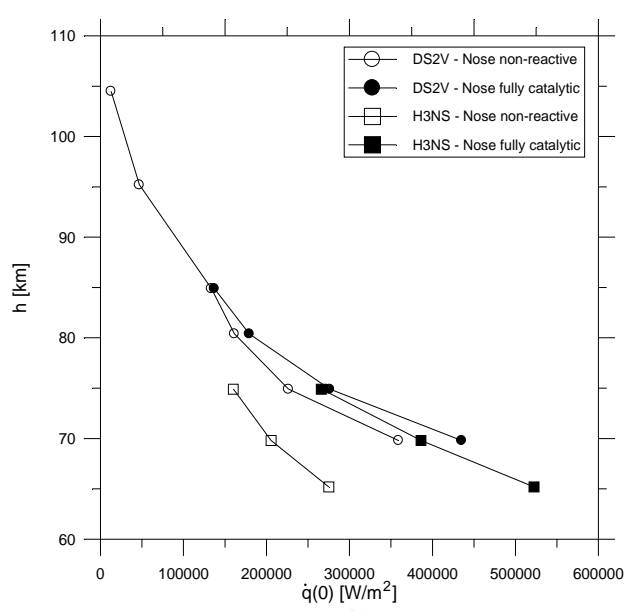

(b)

Figure 5. Comparison of heat flux distributions along the capsule surface (a) and at the stagnation point (b) by DS2V and H3NS

Figures 6a, b show the molar fractions of atomic Oxygen $\left(\alpha_{0}\right)$ and of atomic Nitrogen $\left(\alpha_{N}\right)$ along the surface from the nose stagnation point at $\mathrm{h}=69.8 \mathrm{~km}$. As said before, the lack of recombination equations makes the values of $\alpha_{\mathrm{O}}$ and $\alpha_{N}$ higher for H3NS. This condition implies that a larger amount of energy is spent for dissociation and therefore a lower amount of energy is exchanged with surface at the nose stagnation point. On the opposite, at the 
junction nose-frustum where catalyticity increases from zero to one, H3NS achieves an higher surface recombination and therefore an higher heat flux.

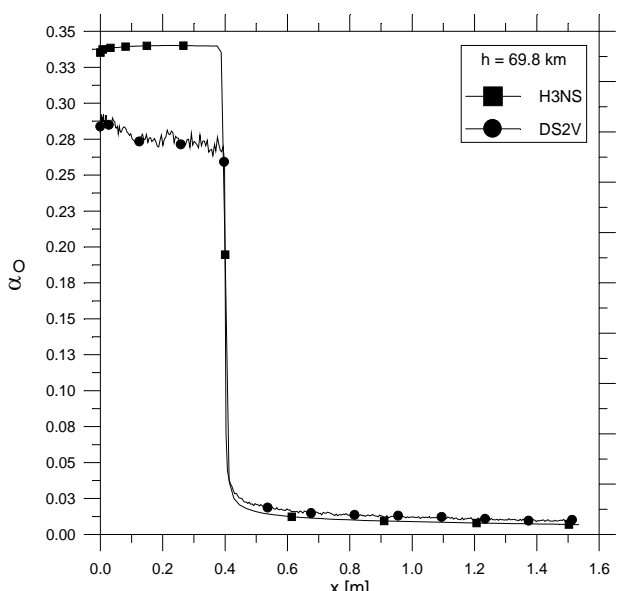

(a)

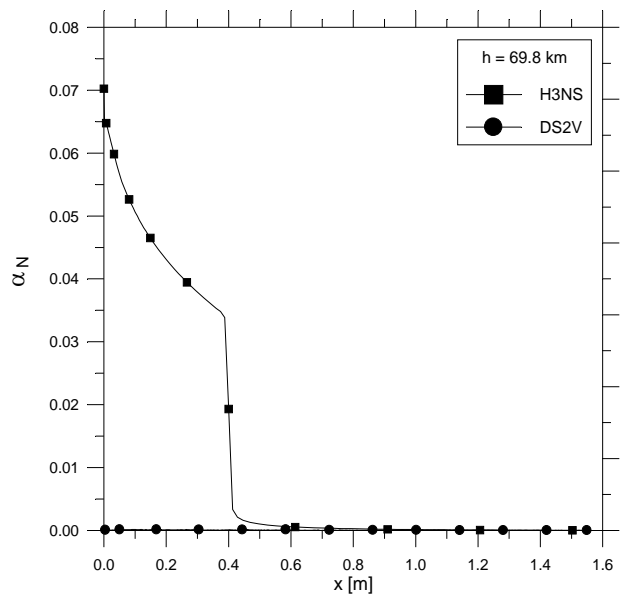

(b)

Figure 6. Molar fractions of atomic Oxygen (a) and atomic Nitrogen (b) along the EXPERT surface at $69.8 \mathrm{~km}$ from H3NS and DS2V

\section{CONCLUSIONS AND FURTHER DEVELOPMENTS}

The data base of the EXPERT capsule has been broadened by computations in the altitude interval 70-105 km by 3$\mathrm{D}$ and 2-D DSMC codes. The work involved aerodynamic parameters (axial and normal force coefficients), longitudinal stability (pitching moment coefficient and fluctuation of position of pressure center along the axis) and heat flux (along the surface and at the nose stagnation point).

The capsule showed good stability behavior in the whole altitude interval up to an angle of attack of about 40 deg.. It has been also verified that, when the capsule is at an angle of attack, the effect of non-symmetry of the flow field is practically irrelevant.

Heat flux was evaluated in the altitude interval $65-75 \mathrm{~km}$ also by a CFD code. The chemical model implemented in the CFD code, lacking of some recombination equations, produced in the flow field, stronger dissociation compared with the one computed by the DSMC code. This probably involved, for the CFD code, lower heat flux at the nose stagnation point, where surface is non-reactive and, at the same time, higher heat flux along the frustum where surface is fully catalytic. To assess this problem, a thermal analysis has been already scheduled by means of the implementation of the same chemical model in both codes.

As the launch of the capsule is currently scheduled in 2011, flight data should be available to verify the results of the present computations, with special regard to the heat flux at the nose stagnation point and along the surface of the capsule.

\section{REFERENCES}

1. A. Schettino, R. Votta, et al. Proc. of the West-East High Speed Flow Field Conference, Moscow, 2007

2. M. Ivanov, P.Vashchenkov, A. Kashkowsky Numerical Investigation of the EXPERT Reentry Vehicle Aerothermodynamics Along the Descent Trajectory, 39 ${ }^{\text {th }}$ AIAA thermophysics Conference, Miami, June 2007

3. G. A. Bird, The DS3V Program User's Guide Ver. 2.5, G.A.B. Consulting Pty Ltd, Sydney, 2006

4. G.Ranuzzi, S.Borreca, H3NS: Code Development Verification and Validation, CIRA-CF-06-1017, Capua, 2006

5. G.A. Bird, The DS2V Program User's Guide Ver. 3.2, G.A.B. Consulting Pty Ltd, Sydney, 2005

6. J.N. Moss, Rarefied Flows of Planetary Entry Capsules, Proceedings of AGARD-R-808, Rhode-Saint-Genèse, 1997

7. G.A Bird., "Molecular Gas Dynamics and Direct Simulation Monte Carlo", Clarendon Press, Oxford (G.B.), 1998

8. G.A. Bird, Sophisticated DSMC, Notes from a Short Course held at the DSMC07 Conference, Santa Fe, USA, 2007

9. C. Park , "A Review of Reaction Rates in High Temperature Air," AIAA Paper 89-1740, June 1989

10. N.M. Kogan, Rarefied Gas Dynamic, Plenum, New York, 1969 